\title{
Polarization properties of a photorefractive Bi12SiO20 crystal and their application in an optical correlator
}

\author{
Edvold, Bent; Andersen, Peter E.; Buchhave, Preben; Petersen, Paul Michael
}

Published in:

I E E E Journal of Quantum Electronics

Link to article, DOI:

10.1109/3.291377

Publication date:

1994

Document Version

Publisher's PDF, also known as Version of record

Link back to DTU Orbit

Citation (APA):

Edvold, B., Andersen, P. E., Buchhave, P., \& Petersen, P. M. (1994). Polarization properties of a photorefractive $\mathrm{Bi} \mathrm{SiO}_{3 \mathrm{~B}}$ crystal and their application in an optical correlator. I E E E Journal of Quantum Electronics, 30(4), 1075-1089. https://doi.org/10.1109/3.291377

\section{General rights}

Copyright and moral rights for the publications made accessible in the public portal are retained by the authors and/or other copyright owners and it is a condition of accessing publications that users recognise and abide by the legal requirements associated with these rights.

- Users may download and print one copy of any publication from the public portal for the purpose of private study or research.

- You may not further distribute the material or use it for any profit-making activity or commercial gain

- You may freely distribute the URL identifying the publication in the public portal 


\title{
Polarization Properties of a Photorefractive $\mathrm{Bi}_{12} \mathrm{SiO}_{20}$ Crystal and their Application in an Optical Correlator
}

\author{
Bent Edvold, Peter E. Andersen, Preben Buchhave, and Paul M. Petersen, Member, IEEE
}

\begin{abstract}
The polarization properties of $\mathrm{Bi}_{12} \mathrm{SiO}_{20}$ (BSO) crystals are investigated in detail theoretically and experimentally, and the results are used to describe the operation of an optical correlator for a particle image velocimeter (PIV) using a BSO crystal as the nonlinear optical element. The work is based on an extension of the optical beam-propagation (OBP) method to include all the significant optical properties of the BSO crystal when used in a two-wave mixing configuration, i.e., optical activity, field-induced birefringence, and anisotropic diffraction. The model is able to handle multiple gratings where the input beams do not have to be symmetric about the axis of propagation. Using the numerical model the polarization properties of the BSO crystal are analyzed and the operation of the correlator is explained. The model is able to take into account self-diffraction effects, and it is shown that these effects can have a significant influence in the setup employed for the optical correlator even when the diffraction efficiency is low. The predictions of the numerical model are verified by extensive experiments on the polarization state of the output of the correlator as a function of operating conditions and of the polarization state of the input beams.
\end{abstract}

\section{INTRODUCTION}

A thorough understanding of the propagation of multiple plane waves through a photorefractive crystal such as $\mathrm{Bi}_{12} \mathrm{SiO}_{20}$ (BSO) and a description of the interaction of these waves as a result of diffraction in the gratings induced by the photorefractive effect is of fundamental importance for the application of photorefraction in optical systems and devices.

In our work we have used a BSO crystal as the active element in an optical autocorrelator for the analysis of particle image velocimetry (PIV) recordings. During this work it became apparent that a deeper understanding of the polarization properties of the waves propagating through the crystal was necessary in order to explain the operation of the correlator. As an example, the intensity of the various peaks in the autocorrelation plane is sensitive to the operating conditions of the crystal such as the applied voltage, holographic fringe spacing, and velocity of the particles. Also the polarization state (polarization angle and ellipticity) of the input beams influences not only the diffraction efficiency, but also the distribution of the diffracted light intensity between the center peak and the side peaks, which make up the signal. By

Manuscript received July 6, 1993. P. M. Petersen's work was supported by the Danish Technical Research Council under Grant Number 5.26.09.19.

B. Edvold was with the Department of Physics, The Technical University of Denmark, 2800 Lyngby, Denmark; he is now with LYCOM A/S, NKT Allé 75, DK-2605 Brondby, Denmark.

P. E. Andersen, P. Buchhave, and P. M. Petersen are with the Department of Physics, The Technical University of Denmark, 2800 Lyngby, Denmark. IEEE Log Number 9215804. adjusting the input polarization it is even possible to obtain a nonsymmetric distribution of light intensity between the side peaks in the correlation plane, a result not normally expected of an autocorrelation function. Finally, as has also been noted by previous workers, the polarization of the center peak may differ from that of the side peaks, a fact that provides an opportunity for an improvement in the signal-to-noise ratio by appropriate polarization filtering in the output plane.

However, these effects are impossible to predict or explain from a simple model of a thin holographic grating, and the complicated nature of the diffraction process in a BSO crystal, which exhibits both field-induced birefringence and optical activity, does not allow an easy, intuitive interpretation of the diffraction process in the thick crystal. Available analytical treatments or numerical models of the diffraction process in BSO do not go far enough to be useful in our situation, which included multiple input beams at arbitrary (but small) input angles. Thus we set out to develop a model for the anisotropic diffraction process in BSO, which would be sophisticated enough to include our problem, i.e., the model should be able to handle multiple gratings inside the BSO crystal, input beams that do not have to be symmetric about the crystal axis, and of course be able to take into account the optical properties of the BSO crystal. Furthermore the model is able to examine the influence of self-diffraction during the writing of the gratings, which enables us to show that self-diffraction can have a significant influence on the diffraction efficiency of the center peak in the optical autocorrelation function.

The numerical model is verified through a comprehensive experimental survey of the polarization properties of the BSO crystal in the configurations relevant for the optical correlator.

\section{A. The Optical Correlator for PIV}

To illustrate the problem we shall briefly explain the application of the BSO crystal in an optical correlator for the analysis of PIV images. In PIV [1], [2] the velocity distribution in a cross section of a flow field is measured by recording the positions of small particles carried with the fluid. In a typical PIV measurement, the particles are illuminated by two intense pulses of laser light with a small time separation from a double-pulsed ruby or Nd-YAG laser. The laser beam is expanded into a thin sheet and the particle positions in the laser sheet at the time of the laser pulses are recorded on a high-resolution photographic film. After development, the film is analyzed point by point (the "point" being an area of the film, the so-called interrogation area, that is small compared to the spatial scales of the velocity fluctuations) to obtain a 
vector plot of the instantaneous velocity field. Under normal operating conditions the film will contain a high concentration of particle images, and each interrogation area will contain a certain number of particle image pairs (of the order of 5 to 20). The task is now to find the average displacement of the particles within each interrogation area irrespective of a possible number of incomplete pairs (single images) and background noise in the film. Once the particle image displacement, represented by the coordinates $\Delta X$ and $\Delta Y$, has been measured, the two-dimensional velocity, $\left(v_{x}, v_{y}\right)$, projection onto the cross section of the flow may be found knowing the magnification $M$ of the imaging optics and the time separation $\Delta t$ between the laser pulses:

$$
v_{x}=\frac{\Delta X}{M \cdot \Delta t}, \quad v_{y}=\frac{\Delta Y}{M \cdot \Delta t}
$$

With more than one image pair present on the average in an interrogation area, the most efficient method for extracting the information about the average image displacement in an interrogation area is to apply the operation of twodimensional autocorrelation to the segment of the image within the interrogation area [3]:

$$
R(\boldsymbol{S})=\int I(\boldsymbol{X}) I(\boldsymbol{X}+\boldsymbol{S}) d \boldsymbol{X}
$$

where $\boldsymbol{X}=(X, Y)$ is the position in the image plane, $\boldsymbol{S}=\left(S_{x}, S_{y}\right)$ is the coordinate in the correlation plane, and $I$ is the image intensity. This operation is implemented by illuminating the interrogation area with a laser or white light source and image the interrogation area onto a matrix detector such as a CCD camera, as illustrated in Fig. 1(a). The video signal is digitized by a so-called frame grabber and stored. Subsequently the autocorrelation is performed digitally, and the velocity direction and magnitude are computed from the position of the autocorrelation peak relative to the center peak. Fig. 1(b) shows a $1 \times 1-\mathrm{mm}$ segment of a PIV image, a typical interrogation area. Fig. 1(c) shows the corresponding 2-D autocorrelation. The average displacement is indicated by $\Delta X$ and $\Delta Y$.

In practice the autocorrelation is not computed directly, but through a dual application of a 2-D fast Fourier transform (FFT) in accordance with the Wiener-Khintchine theorem:

$$
R(\boldsymbol{S})=\mathbf{F}^{-1}\left\{|\mathrm{~F}\{I(\boldsymbol{X})\}|^{2}\right\}
$$

where $\mathrm{F}$ denotes Fourier transformation. Still, this process is time consuming, and since a PIV image may be resolved in several thousand interrogation areas the processing of a single PIV image may take hours or even days on a PC-type computer. As an example, the processing time on a $386 \mathrm{PC}$ with an additional 8 MFLOP array processor plug-in board is about 20 s per velocity vector, resulting in a processing time of 55 hours for a single PIV image with 10000 vectors.

An optical correlation processor has the potential of much higher speed. Fig. 2(a) shows a BSO-based optical correlator [3]-[6], which has been implemented in our laboratory. If the BSO is just considered a component, which is able to store a thin hologram, the analysis is simple: Lens $\mathrm{L}_{1}$ acts as a Fourier transform lens placing the Fourier transform $A$ of the

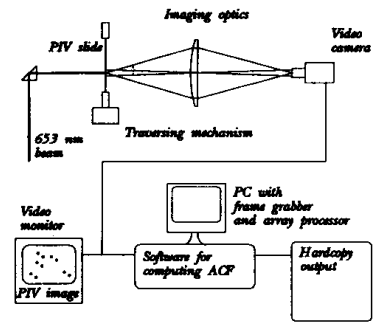

(a)

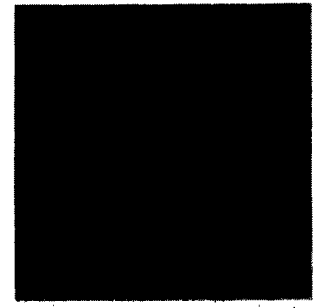

(b)

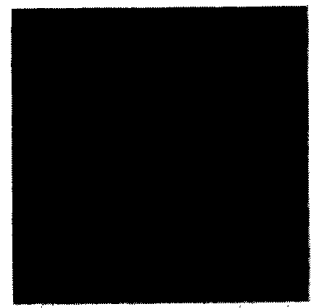

(c)
Fig.' 1. (a) Setup for numerical processing of a PIV image. (b) Closeup of a PIV image (interrogation area size $1 \times 1 \mathrm{~mm}^{2}$ ). (c) The numerical autocorrelation function.

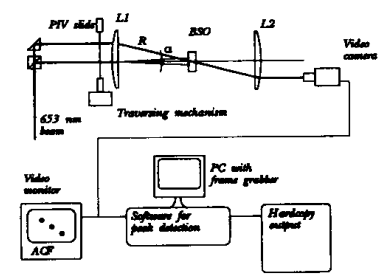

(a)

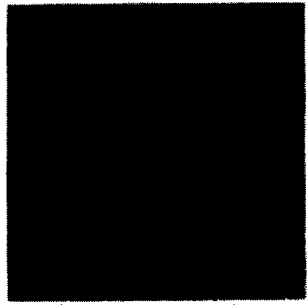

(b)

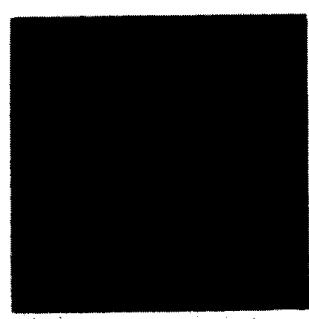

(c)
Fig. 2. (a) Setup for optical processing of a PIV image. (b) Closeup of a PIV image (interrogation area size $1 \times 1 \mathrm{~mm}^{2}$ ). The interrogation area is identical to the one in Fig. 1(b). (c) The optical autocorrelation function.

object (the interrogation area of the PIV transparency) in the crystal. This field is recorded as a hologram in the crystal by the photorefractive effect using the plane-wave reference beam $R$ to create interference fringes in the crystal. In the simple theory the crystal is considered to be sensitive to the intensity of the optical field, which creates spatial changes in the refractive index of the crystal. The hologram is then present as a weak phase modulation proportional to the square of the total optical field. The transmittance of the crystal may thus be expressed by:

$$
t=e^{i \beta I} \cong 1+i \beta I
$$


where $\beta$ is a measure of the nonlinear response of the crystal and $I$ is the intensity:

$$
I=|A+R|^{2}=|A|^{2}+|R|^{2}+A^{*} R+A R^{*}
$$

Blocking the reference beam, the hologram may be read out by means of the Fourier transformed object beam $A$ resulting in an output from the crystal consisting of the following terms:

$$
\begin{aligned}
U & =A t=A(1+i \beta I) \\
& =A+i \beta\left\{A|A|^{2}+A|R|^{2}+A A^{*} R+A A R^{*}\right\}
\end{aligned}
$$

This output is again Fourier transformed by the lens $L_{2}$, resulting in the output field:

$$
\begin{aligned}
u= & \mathrm{F}\{U\}=\mathrm{F}\{A\}+i \beta \mathrm{F}\left\{A|A|^{2}\right\}+i \beta \mathrm{F}\left\{A|R|^{2}\right\} \\
& +i \beta \mathrm{F}\left\{A A^{*} R\right\}+i \beta \mathrm{F}\left\{A A R^{*}\right\}
\end{aligned}
$$

The desired output is the fourth term, which will propagate in the direction of the reference beam. This term represents a convolution of the reference beam $R$ with the autocorrelation of the object beam $A$. If the reference beam is a plane wave we thus write:

$$
u_{4}=i \beta a \otimes a
$$

where $\otimes$ denotes the autocorrelation. The subsequent procedure is now to measure the distance between the center peak and the correlation peak in the correlation plane, e.g., by means of a video camera and a frame grabber, and to convert this measurement to velocity information. Figs. 2(b) and 2(c) show the interrogation area and the corresponding output from the optical correlator.

As mentioned, the simple analysis in (4)-(8) is convenient for explaining the Fourier optical function of the correlator, but it is not able to explain the detailed behavior of the BSO crystal in the correlator, and a more detailed analysis taking into account the optical properties of the BSO crystal is needed. As an example of a phenomenon that the simple analysis is not able to explain, we refer to Fig. 3 showing a correlation function obtained with our experimental setup. This figure shows an autocorrelation that is clearly asymmetric: The two side peaks have noticeably different intensities. Furthermore, Fig. 4 shows a correlation that has different polarization states in the center peak as opposed to the two side peaks. This is demonstrated in the lower part of the figure, where a linear polarization filter has been inserted in the output beam. The transmission shows that the center peak is nearly linearly polarized, whereas the side peaks are nearly circularly polarized.

\section{B. Optical Wave Propagation in BSO}

The propagation and diffraction of optical plane waves in a thick holographic grating was analyzed in the now classical paper on coupled wave equations in isotropic media by Kogelnik [7]. However, the equivalent problem in BSO is much more complex due to the anisotropic diffraction and the concomitant field-induced birefringence and optical activity in that crystal. Moreover, in order to analyze the function of the optical correlator outlined above it is necessary to include

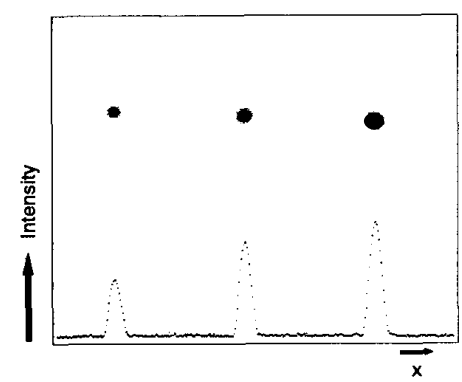

Fig. 3. Example of an asymmetric autocorrelation function. The dotted curve represents the intensity distribution between the three peaks.

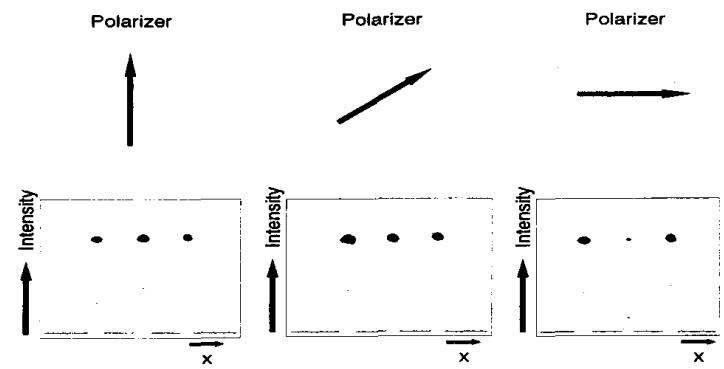

Fig. 4. Example of autocorrelation functions, when a polarizer is inserted in the output plane. The dotted curve represents the intensity distribution between the three peaks, and the arrows above represent the orientation of the polarizer. (a) Polarizer orientation $0^{\circ}$ with respect to the vertical. (b) Polarizer orientation $45^{\circ}$ with respect to the vertical. (c) Polarizer orientation $90^{\circ}$ with respect to the vertical.

multiple input beams, resulting in multiple gratings slanted with respect to the axis of propagation. This is very important in an analysis of the optical correlator as the correlator can be explained by diffraction in three gratings, as will be shown in Section III. Finally it is essential to be able to analyze the read-out process not just at the Bragg angle, but as a function of the Bragg detuning angle, i.e., at small angular deviations from the nominal Bragg angle.

In addition to these essential requirements to a solution, we would also like to be able to predict the effect of selfdiffraction, i.e., the modification of the grating occurring throughout the crystal because of the interference of the writing beams with the diffracted beams themselves. Moreover, most solutions described in the literature assume weak interaction (which is a good approximation in the case of BSO) and small intersection angles (fields normal to the average propagation direction). The latter approximation simplifies the analysis, but precludes the study of the effect of the axial component of the electric field vector, and the possible effect of a misalignment of the crystal axes with respect to the axis of propagation.

The coupled wave equations describing optical wave propagation in BSO may be solved analytically in special cases. Petrov et al. [8] found analytical solutions to the case of only field-induced birefringence and anisotropic diffraction, while Pencheva et al. [9] analyzed the case of diffraction in a medium possessing optical activity and showed the angular Bragg detuning of the optimum diffraction efficiency. Kuhktarev [10] describes the formation of holographic gratings 
in BSO, including both field-induced birefringence and optical activity. However, the work by Vachss and Hesselink [11] comes closest to solving our problem as described above. Vachss and Hesselink's solution includes both field-induced birefringence and optical activity, and the read-out process is analyzed with respect to Bragg detuning. Still, their solution describes the diffraction process involving only a single fixed grating in the crystal, which prevents this theory from explaining the multiwave-coupling in the autocorrelator. Assuming a fixed grating also excludes the effects of self-diffraction. However, their analysis should be applicable to the more general problem of a single slanted grating.

Another approach is to solve the system of coupled firstorder differential equations resulting from a direct solution of Maxwell's equations. A rigorous, vectorial solution has been developed by Glytsis and Gaylord [12]. This solution also applies to multiple superimposed gratings, and should in principle apply to the correlator problem. However, the examples presented by the authors do not explicitly include BSO with concomitant field-induced birefringence and optical activity, nor is it possible to explicitly see the effect of selfdiffraction (the grating is assumed constant throughout the crystal).

An integral approach was used by Mallick et al. [13] who also included field birefringence and optical activity. These authors arrive at an analytic expression for the polarization of the directly transmitted beams and calculate the polarization properties of the diffracted beams. However, these authors used a standard integration routine to find directly the field at the exit of the grating, and thus were not able to include self-diffraction. Unfortunately, the solution derived in their paper is also limited by including only one (fixed) grating and, furthermore, Bragg detuning during the read-out process is not included in their analysis.

We have used a fourth approach to the solution of the diffraction problem in BSO, the so-called optical beam propagation (OBP) method [14]. In this method the crystal is divided into a number of thin, parallel slabs. In each slab the gratings are defined by the field incident on the slab. Subsequently the incident field is allowed to interact with the slab, both in terms of the bulk electrooptic properties including field-induced birefringence and optical activity and in terms of diffraction in multiple gratings written by the incident beams. By working from slab to slab from the front surface of the grating to the exit surface and by including a large enough number of slabs, the method converges towards a stable solution to the wave propagation problem. The method is able to include all effects mentioned above, including input beams which are nonsymmetric with respect to the crystal axis and self-diffraction effects. Our method is an extension of the previous work by Marrakchi, Johnson, and Tanguay [14]-[16] by including multiple slanted gratings with Bragg detuning and self-diffraction.

\section{Organization of this Paper}

In this paper we present in Section II the derivation of the permittivity matrix representing the bulk optical properties of the BSO crystal, and the set of coupled difference equations describing the interaction of the incident fields with multiple gratings in the crystal. Also in Section II we describe the practical implementation of the model as a computer program running on a personal computer. We present the convergence properties of the model and the computation time needed.

Section III describes experimental results, which illustrate the validity of the model. We show close agreement between the polarization properties such as polarization angle and ellipticity of the diffracted beams computed by the model, and the polarization properties measured in a series of experiments using various polarization states, including linear and elliptical polarization of the incident beams. The effect of self-diffraction is analyzed and shown to have a significant influence on the center peak in the autocorrelation. We then present some applications of the model to explain the performance of the optical PIV correlator. The model is able to explain the asymmetry of the correlation peaks in agreement with the experimental results. The model may also be used to select the optimum crystal thickness and applied electric field for optimum performance of the correlator. Finally, we describe some problems that may be envisioned with the application of the BSO correlator for the processing of turbulent velocity data.

\section{THE NumERICAL MODEL}

Before we proceed with the derivation of our numerical model we shall consider the fundamental physical and optical properties of the BSO crystal.

\section{A. Fundamental Physics of the BSO Crystal}

When a photorefractive crystal is illuminated with an interference pattern, mobile charge carriers will propagate toward the dark areas of the crystal and give rise to a space-charge field inside the crystal. The fundamental first-order spatial harmonic component of the space-charge field is calculated from Hall et al. [17]:

$$
E_{s c}=E_{A}+E_{G} \cos \left(\boldsymbol{k}_{G} \cdot \boldsymbol{r}+\phi\right)
$$

Here $E_{A}$ is the electric dc field applied to the crystal, $\boldsymbol{k}_{G}$ denotes the grating vector, $r$ is a coordinate vector, and $\phi$ is the spatial phase shift between the interference pattern and the index grating. The strength of the modulation (linear in modulation approximation [17]) is given by [15]:

$$
\begin{aligned}
E_{G} & =-m E_{s}\left[\frac{E_{A}^{2}+E_{D}^{2}}{E_{A}^{2}+\left(E_{D}+E_{s}\right)^{2}}\right]^{1 / 2} \\
\phi & =\operatorname{Arctan}\left[\left(\frac{E_{D}}{E_{A}}\right)\left(1+\frac{E_{D}}{E_{s}}+\frac{E_{A}^{2}}{E_{D} E_{s}}\right)\right]
\end{aligned}
$$

Here $m$ is the modulation depth of the interference pattern, $E_{s}$ is a saturation field, and $E_{D}$ is the diffusion field. The saturation and diffusion fields are given by:

$$
\begin{aligned}
E_{s} & =\frac{e N_{A}}{\epsilon_{d c} \epsilon_{0} k_{G}} \\
E_{D} & =\left(\frac{k_{B} T}{e}\right) k_{G}
\end{aligned}
$$




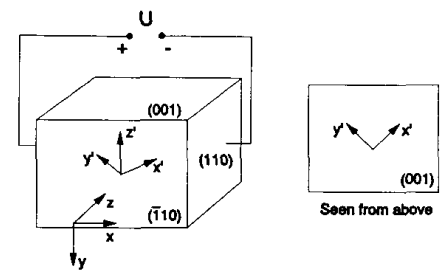

Fig. 5. BSO crystal in the $\boldsymbol{k}_{G} \perp\langle 001\rangle$ configuration.

In these equations $e$ is the electronic charge, $\epsilon_{d c}$ the $\mathrm{dc} \mathrm{di}-$ electric constant, $\epsilon_{0}$ the vacuum permittivity, $k_{B}$ Boltzmann's constant, $T$ the absolute temperature and $N_{A}$ the acceptor concentration inside the crystal.

When the modulation depth is high, the strength of the higher-order spatial harmonics of the space-charge field can be substantial. However, since the higher spatial harmonics are normally not Bragg-matched to the incoming beams, the effects of these components is neglected. In our analysis the modulation depth is low and thus the space-charge field is well approximated by the linear in modulation approximation.

The optical properties of the BSO crystal depend strongly on the orientation of the crystal. The common configuration of the crystal in an autocorrelator setup is the so-called $\boldsymbol{k}_{G} \perp\langle 001\rangle$ configuration, which is shown in Fig. 5. In this configuration the modulated axes are inclined $45^{\circ}$ with respect to the light coordinate system, and the two axes are modulated with opposite signs. In the presence of optical activity the optical properties for this configuration are described by the following permittivity matrix, as propagation is taken to be in the $z$-direction [18]:

$$
\underline{\underline{\epsilon}}=\left\{\begin{array}{cc}
n_{0}^{2}+i 2 \alpha n_{0} / k_{0} & i a-\Delta \epsilon \\
-i a-\Delta \epsilon & n_{0}^{2}+i 2 \alpha n_{0} / k_{0}
\end{array}\right\}
$$

Here $n_{0}$ is the bulk refractive index, $\Delta \epsilon=n_{0}^{4} r_{41} E_{s c}$ includes field-induced birefringence and modulation, $r_{41}$ the electrooptic tensor value for BSO, and $a$ is given by the optical rotatory power $\rho$ through

$$
a=\frac{2 n_{0} \rho}{k_{0}}
$$

where $k_{0}=2 \pi / \lambda_{0}$ denotes the vacuum wave number.

In addition to [18] we have in (14) included the optical absorption $\alpha$.

\section{B. Derivation of the Model}

In deriving the numerical model we assume all optical fields to be plane waves of the form:

$$
E_{T}=E_{i}\left(z_{i}\right) e^{i \boldsymbol{k}_{i} \cdot \boldsymbol{r}}
$$

where $\boldsymbol{r}=(x, y, z)$ and $\left|\boldsymbol{k}_{i}\right|=\left|n_{0} \boldsymbol{k}_{0}\right|=k$ is the wave number in the crystal. $z_{i}=z / \cos \theta_{i}$ is a coordinate measured along the direction of propagation of ray $i$. The plane wave given in (16) depends only on the coordinate $z_{i}$ and thus has no variation transverse to the direction of propagation. At this point the total field $E_{T}$ consists only of a single wave, but

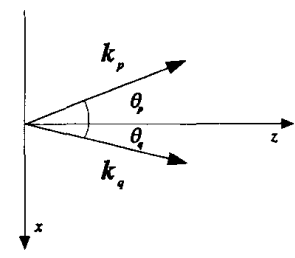

Fig. 6. Coordinate system used when deriving the numerical model.

later the propagation of several waves will be examined. In the coordinate system shown in Fig. $6, \boldsymbol{k}_{i}$ is given by:

$$
\boldsymbol{k}_{i}=k\left(\sin \theta_{i} \hat{\boldsymbol{x}}+\cos \theta_{i} \hat{\boldsymbol{z}}\right) .
$$

In (16) the phase factor is given by the isotropic refractive index of the crystal. This way we include all anisotropic characteristics of the BSO crystal in the complex field vector $E_{i}\left(z_{i}\right)$. By using this approach optical activity, field-induced birefringence and anisotropic diffraction are considered perturbations to the isotropic wave propagation. The effect of these perturbations will appear as a $z_{i}$-dependence of both the amplitude and the phase of the complex field vectors.

To study the propagation of these waves through the crystal, we insert the field from (16) in the time-independent wave equation (see, e.g., [14]):

$$
\nabla^{2} E_{T}+k_{0}^{2} \underline{\underline{\epsilon}} E_{T}=0
$$

yielding

$$
\begin{array}{r}
\left(\nabla^{2} E_{i}+2 i k\left(\frac{\partial E_{i}}{\partial x} \sin \theta_{i}+\frac{\partial E_{i}}{\partial z} \cos \theta_{i}\right)\right. \\
\left.+\left(k_{0}^{2} \underline{\underline{\epsilon}}-k^{2} \underline{\underline{I}}\right) E_{i}\right) e^{i k\left(x \sin \theta_{i}+z \cos \theta_{i}\right)}=0
\end{array}
$$

where $\underline{I}$ is the unity matrix. As explained in the previous section, the complicated optical properties of the BSO crystal are included in the permittivity tensor. Hence, (19) describes the propagation of one ray through the BSO crystal in the presence of optical activity, field-induced birefringence, and photorefractive modulation of the refractive index. For well-collimated rays we use the slowly varying envelope approximation (SVEA) and neglect the first term in (19). Furthermore we will rewrite the second term as follows

$$
\frac{\partial E_{i}}{\partial x} \sin \theta_{i}+\frac{\partial E_{i}}{\partial z} \cos \theta_{i}=\frac{d E_{i}}{d z_{i}} .
$$

Thus (19) may be written:

$$
\left(2 i k \frac{d E_{i}}{d z_{i}}+\left(k_{0}^{2} \underline{\underline{\epsilon}}-k^{2} \underline{\underline{I}}\right) E_{i}\right) e^{i k\left(x \sin \theta_{i}+z \cos \theta_{i}\right)}=0
$$

In the derivation of (21) we have maintained the phase of the ray throughout the calculations. When only one wave propagates through the crystal or if the waves propagate symmetrically about the axis of propagation [14] this is not necessary since the phase term can be removed by reduction of (21). However, when several waves propagate through the crystal, each ray will have a different phase, and hence the phase terms can no longer be left out. 
In the general case we write the field distribution as

$$
E_{T}=\sum_{i} E_{i}\left(z_{i}\right) e^{i k\left(x \sin \theta_{i}+z \cos \theta_{i}\right)}
$$

Inserting this field distribution into the wave equation, (21), yields

$$
\sum_{i}\left(2 i k \frac{d E_{i}}{d z_{i}}+\left(k_{0}^{2} \underline{\underline{\epsilon}}-k^{2} \underline{\underline{I}}\right) E_{i}\right) e^{i k\left(x \sin \theta_{i}+z \cos \theta_{i}\right)}=0
$$

This is the fundamental equation for the propagation of several beams through the crystal. To solve (23) we use the following procedure: The equation is divided into subsets of equations by grouping waves, which propagate in the same direction. Each subequation is then solved separately. The matching of waves results in a phase condition, which will be discussed in more detail later on.

Because of diffraction in the grating, there will normally be several rays propagating in the same direction. As may be seen from (23), the total contribution of diffracted rays to a wave propagating in a particular direction is found by superposition of the contributions from each individual diffracted wave. Thus, to find the form of the total contribution we need only consider the case of two waves, i.e.

$$
E_{T}=E_{p}\left(z_{p}\right) e^{i \boldsymbol{k}_{p} \cdot \boldsymbol{r}}+E_{q}\left(z_{q}\right) e^{i \boldsymbol{k}_{q} \cdot \boldsymbol{r}}
$$

where we will consider diffraction of ray $q$ into ray $p$. In this case (23) yields

$$
\begin{aligned}
2 i k[ & \left.\frac{d E_{p}}{d z_{p}} e^{i \boldsymbol{k}_{p} \cdot \boldsymbol{r}}+\frac{d E_{q}}{d z_{q}} e^{i \boldsymbol{k}_{q} \cdot \boldsymbol{r}}\right] \\
& +\left(k_{0}^{2} \underline{\underline{\epsilon}}-k^{2} \underline{\underline{I}}\right)\left[E_{p} e^{i \boldsymbol{k}_{p} \cdot \boldsymbol{r}}+E_{q} e^{i \boldsymbol{k}_{q} \cdot \boldsymbol{r}}\right]=0 .
\end{aligned}
$$

In order to collect terms with the same phase we need to study how the permittivity tensor alters the phases of the individual waves. The permittivity matrix for the $\boldsymbol{k}_{G} \perp\langle 001\rangle$ orientation of the BSO crystal is given in Section II.A. In the following discussion it will, however, be advantageous to split the permittivity tensor into two parts, one containing the bulk properties of the crytal and one containing the diffraction contribution:

$$
\underline{\underline{\epsilon}}=\underline{\underline{\epsilon}}^{(1)}+\underline{\underline{\epsilon}}^{(2)}
$$

with

$$
\underline{\underline{\epsilon}}^{(1)}=\left\{\begin{array}{cc}
n_{0}^{2}+i 2 \alpha n_{0} / k_{0} & i a-n_{0}^{4} r_{41} E_{A} \\
-i a-n_{0}^{4} r_{41} E_{A} & n_{0}^{2}+i 2 \alpha n_{0} / k_{0}
\end{array}\right\}
$$

and

$$
\left.\begin{array}{lc}
\underline{\epsilon}^{(2)}= & \\
0 & -n_{0}^{4} r_{41} E_{G} \cos \left(\boldsymbol{k}_{G} \cdot \boldsymbol{r}+\phi\right) \\
-n_{0}^{4} r_{41} E_{G} \cos \left(\boldsymbol{k}_{G} \cdot \boldsymbol{r}+\phi\right) & 0
\end{array}\right\}
$$

The influence of the refractive index modulation on the phase of the electric field becomes clear as the cosine function is written in complex notation:

$$
\cos \left(\boldsymbol{k}_{G} \cdot \boldsymbol{r}+\phi\right)=\frac{1}{2}\left[e^{i\left(\boldsymbol{k}_{G} \cdot \boldsymbol{r}+\phi\right)}+e^{-i\left(\boldsymbol{k}_{G} \cdot \boldsymbol{r}+\phi\right)}\right]
$$

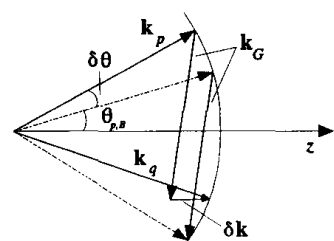

Fig. 7. Definition of phase deficit $\delta \boldsymbol{k}$ caused by anisotropic diffraction.

We may now derive the phase condition for waves propagating in the same direction. As seen in Fig. 7 waves progating in an off-Bragg direction indicated by the angle $\theta_{B}+\delta \theta$ suffer a phase deficit determined by the wave vector difference $\delta \boldsymbol{k}$. This phase deficit will later influence the wave amplitude. In accordance with [15] we assume $\delta \boldsymbol{k}$ to be directed in the $z$-direction. We thus find for the phase matching condition:

$$
\boldsymbol{k}_{p}=\boldsymbol{k}_{q} \pm \boldsymbol{k}_{G}+\delta \boldsymbol{k}
$$

with $\left|\boldsymbol{k}_{p}\right|=\left|\boldsymbol{k}_{q}\right|=k$ and $\delta \boldsymbol{k}$ in the direction of the $z$-axis.

We may use the projection onto the $x$-axis to find the direction of the diffracted wave:

$$
k \sin \theta_{p}=k \sin \theta_{q} \pm k_{G} \sin \theta_{G}
$$

From the projection onto the $z$-axis we find the size of the detuning parameter $\delta k$ :

$$
\delta k=k \cos \theta_{p}-k \cos \theta_{q} \pm k_{G} \cos \theta_{G}
$$

As we are working in a thin slab near Bragg diffraction, each wave is assumed to give rise to only two diffraction orders. However, as we later divide the crystal into thin slabs, these diffracted waves will couple into higher orders as the beam progresses through the crystal. For the moment we shall consider only the plus term in (30). However, the calculations for the other sign are completely analogous. The resulting equation may then be obtained from (25) using the phase conditions (31) and (32):

$$
2 i k \frac{d E_{p}}{d z_{p}}+\left(k_{0}^{2} \underline{\underline{\epsilon}}^{(1)}-k^{2} \underline{\underline{I}}\right) E_{p}+k_{0}^{2} \underline{\underline{\epsilon}}^{\left(2^{\prime}\right)} E_{q} e^{i \delta k z}=0
$$

where $\underline{\epsilon}^{\left(2^{\prime}\right)}$ now contains only one phase factor:

$$
\underline{\underline{\epsilon}}^{\left(2^{\prime}\right)}=\left\{\begin{array}{cc}
0 & -\frac{1}{2} n_{0}^{4} r_{41} E_{G} e^{i \phi} \\
-\frac{1}{2} n_{0}^{4} r_{41} E_{G} e^{i \phi} & 0
\end{array}\right\}
$$

We have now split the time independent wave equation into a series of simple differential equations of the form of (33). These equations are, however, coupled, and since we wish to include self-diffraction effects, the phase factor $e^{i \phi}$ may not be constant throughout the crystal. We therefore wish to find some way of decoupling the equations, and in order to do this we apply the so-called optical beam propagation method [14]. In this approach the crystal is divided into a number of slabs as shown in Fig. 8. If the number of slabs is sufficiently high, the differential equations may be decoupled by treating diffraction and the effects of absorption, optical activity, 


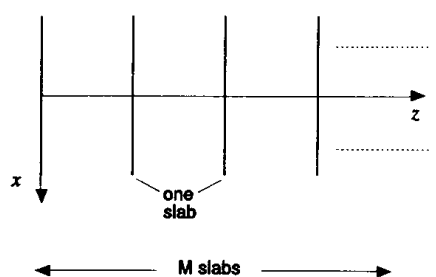

Fig. 8. Division of the BSO crystal into slabs.

and birefringence as independent contributions. Writing the components explicitly, (33),

$$
\begin{aligned}
& \frac{d}{d z_{p}}\left\{\begin{array}{l}
E_{p, x} \\
E_{p, y}
\end{array}\right\}= \\
& \left\{\begin{array}{cc}
-\alpha & -\rho-i \frac{1}{2} k n_{0}^{2} r_{41} E_{A} \\
\rho-i \frac{1}{2} k n_{0}^{2} r_{41} E_{A} & -\alpha
\end{array}\right\}\left\{\begin{array}{l}
E_{p, x} \\
E_{p, y}
\end{array}\right\} \\
& +\left\{\begin{array}{cc}
0 & -i \frac{1}{4} k n_{0}^{2} r_{41} E_{G} e^{i \phi} \\
i \frac{1}{4} k n_{0}^{2} r_{41} E_{G} e^{i \phi} & 0
\end{array}\right\}\left\{\begin{array}{l}
E_{q, x} \\
E_{q, y}
\end{array}\right\} e^{i \delta k z}
\end{aligned}
$$

The first term in (35) shows how the ray is altered by itself by optical activity and field induced birefringence, whereas the second term describes the diffraction of wave $q$ into the direction of wave $p$. The field amplitude $\boldsymbol{E}_{\boldsymbol{p}}^{\text {out }}$ after propagation through the $i$ th slab ranging from $z$ to $z+\Delta z$, is then found from:

$$
\boldsymbol{E}_{\boldsymbol{p}}^{\text {out }}=\boldsymbol{E}_{\boldsymbol{p}}^{\boldsymbol{i n}}+\Delta_{p p}+\Delta_{q p}
$$

where $\boldsymbol{E}_{\boldsymbol{p}}^{\boldsymbol{i n}}$ is the field amplitude at the entrance of the $i$ th slab, $\Delta_{p p}$ is the contribution from ray $p$ to itself, and $\Delta_{q p}$ is the contribution from ray $q$ to ray $p$. The contributions $\Delta_{p p}$ and $\Delta_{q p}$ are easily found, if (35) is decoupled assuming constant field amplitudes $\boldsymbol{E}_{\boldsymbol{p}}$ and $\boldsymbol{E}_{\boldsymbol{q}}$ through each slab:

$$
\begin{aligned}
& \Delta_{p p}= \\
& \frac{\Delta z}{\cos \theta_{p}}\left\{\begin{array}{cc}
-\alpha & -\rho-i \frac{1}{2} k n_{0}^{2} r_{41} E_{A} \\
\rho-i \frac{1}{2} k n_{0}^{2} r_{41} E_{A} & -\alpha
\end{array}\right\}\left\{\begin{array}{l}
E_{p, x} \\
E_{p, y}
\end{array}\right\}
\end{aligned}
$$

$\Delta_{q p}=$

$$
\begin{aligned}
& \frac{1}{\cos \theta_{p}} \frac{e^{i \delta k z}}{i \delta k}\left(e^{i \delta k \Delta z}-1\right) \\
& \cdot\left\{\begin{array}{cc}
0 & -i \frac{1}{4} k n_{0}^{2} r_{41} E_{G} e^{i \phi} \\
i \frac{1}{4} k n_{0}^{2} r_{41} E_{G} e^{i \phi} & 0
\end{array}\right\}\left\{\begin{array}{l}
E_{q, x} \\
E_{q, y}
\end{array}\right\}
\end{aligned}
$$

These two equations are the fundamental equations in our model describing the propagation and diffraction through the BSO crystal. As mentioned above, the derivation is performed assuming two rays propagating through the crystal and considering only one of the two fundamental diffraction orders. However, since all diffraction contributions in one particular direction can be superimposed, the overall diffraction contribution per slab is easily computed by substituting the grating parameters and the field values for ray $q$ in (38). Analogously, diffraction from the second fundamental diffraction order is found by altering the sign corresponding to the second term in (29).
In each slab we calculate only the two fundamental diffraction orders. However, as the crystal is divided into many slabs, higher-order diffraction orders will automatically be formed if the diffraction contributions from each slab add up in phase throughout the crystal. Thus the model can also be used to investigate higher diffraction orders. In a thick crystal, however, the phase-matching conditions will normally allow propagation of only the first diffraction order.

When several gratings are present in the crytal we write the total space-charge field as a sum of the individual grating components:

$$
E_{s c}=E_{A}+\sum_{i} E_{G, i} \cos \left(\boldsymbol{k}_{G, i} \cdot \boldsymbol{r}+\phi_{i}\right)
$$

Using this space charge field the diffraction contribution from each slab is still computed from (38).

The phase-matching conditions: After having derived the fundamental formulas for calculating the diffraction contributions for one slab we will return to the discussion of the influence of the phase-matching conditions given by (31) and (32). It is clear that these conditions are quite analogous to the Bragg condition for isotropic diffraction in a thick holographic grating. However, it is interesting how these conditions apply to the case of anisotropic diffraction. The important parameter in this discussion is the phase detuning parameter $\delta k$ given in (32). As seen from (38) this parameter has a direct influence on the phase of the diffracted ray from a given slab. When the read-out beam is perfectly Bragg-matched to the grating inside the crystal the phase detuning is zero and the phase terms in (38) cancel out. However, in the presence of optical activity and in the case of anisotropic diffraction this does not imply that the diffraction contributions from all slabs have identical phases. As is seen from (37) and (38), the presence of optical activity and field induced birefringence alters the phase of the complex field vectors whereby the diffraction contributions from different slabs generally do not add up in phase. In the case of a relatively low diffraction efficiency this fact results in a lower diffraction efficiency at the Bragg angle as compared to a corresponding isotropic diffraction situation. In the case of anisotropic diffraction the diffraction efficiency is often increased by detuning the read-out beam slightly from the Bragg angle. In this case the phase matching parameter $\delta k$ becomes non-zero and the phase terms in (38) become important. By applying a slight detuning these phase terms can to some extent balance out the phase mismatch of the different diffraction contributions due to optical activity and field-induced birefringence resulting in a better phase matching of the individual contributions and thus in a higher diffraction efficiency. However, when the detuning becomes too large the phase term $\exp \{i \delta k z\}$ in (38) will vary rapidly, resulting in total phase mismatch of the diffraction contributions from the individual slabs and hence in a negligible total diffraction.

\section{Practical Implementation of the Model}

In the previous section we have discussed the derivation of our numerical model. We have shown how the diffraction problem is solved by the OBP method in the case of two rays propagating through a slab. In this section we will explain 
how the expressions given in (37) and (38) are used to obtain the overall diffraction for a BSO crystal when several rays are propagating and several gratings are present in the crystal. This is done by solving the diffraction problem slab by slab, thereby following each ray through the crystal. When the diffraction contributions for each slab have been calculated they are added to the field vectors of rays with the same propagation angle, carefully taking their phases into account.

In more detail the computations are as follows: When the gratings inside the crystal have been determined, the read-out field must be defined. A given polarization state of the read-out beam is represented as a difference in magnitude and phase of the $x$ - and $y$-components of the electrical field vector. When several rays are used for read-out, the propagation angle and electrical field vector of each ray must be specified. The crystal is then divided into a number of slabs. Starting with slab 1 we first consider birefringence and optical activity using the field vector values at the entrance face of the slab in (37). The contributions found from (37) are added to the field vector values at the entrance face to obtain the field vector values at the exit face of the slab. The next step is to compute the contributions from diffraction of each ray in each grating. This is done by determining the wave vectors of the diffracted rays from (31) and (32) and then inserting the field values at the entrance face in (38) to determine the diffraction contributions. These contributions are now added to the field vector values at the exit face of the slab. When these steps have been completed the diffraction problem has been solved for the first slab, and the new field vectors are now used as input values to the next slab and the procedure described above is repeated for the remaining slabs. However, if this procedure is followed strictly, it is clear that the number of diffraction orders per ray increases by two after each slab. For thick crystals, however, it is well known that only a few diffraction orders will have a significant diffraction efficiency. To prevent the use of valuable calculation time on insignificant diffraction orders, a criterion must therefore be applied to neglect insignificant diffraction orders, thereby speeding up the calculations. An effective and intuitive criterion is applied using the following argument: In a thick grating only rays obeying the Bragg-condition are diffracted, thus giving rise to one single diffraction order. In the OBP model only the diffraction contributions for the first-order diffraction will approximately add in phase, all higher-order diffraction contributions will vary in phase through the crystal, resulting in a very low or negligible diffraction efficiency. Therefore rays for which the intensity is smaller than some fraction of the smallest input intensity are neglected. In order to ensure that not all diffraction orders are neglected, propagation through the first 5-10\% of the slabs should be calculated without neglecting any rays. The actual size of this fraction depends, of course, on the specific diffraction problem and is determined from estimates of the total diffraction efficiency. The optimum value is found by comparing precision and calculation speed for different values.

\section{Self-Diffraction}

At this point we have not yet discussed how the gratings inside the crystal are determined. Normally gratings are determined from the incoming rays and they are assumed to be uniform throughout the crystal. During the writing process however, the field vector of the writing beams changes throughout the crystal due to absorption, birefringence, optical activity, and diffraction of the beams in their own gratings. This is known as self-diffraction, which in some situations causes a significant change in diffraction efficiency. Normally self-diffraction effects are neglected in analytical models of anisotropic diffraction due to their complex nature. In our model, however, self-diffraction effects are easily included. This is achieved by calculating the modulation depth and space-charge field of each grating at the entrance face of each slab. In this manner the gratings are not fixed throughout the crystal, but are determined dynamically, allowing them to be modified throughout the crystal.

In more detail, the calculations for each slab must be expanded to be able to alter the gratings throughout the crystal. To show how this is done it is illustrative to go through the calculations in a slab when self-diffraction is included: first the reference, signal, and read-out fields must be specified in order to determine the gratings inside the first slab. Next the effects of optical activity and field induced birefringence on the read-out beam are calculated using (37), and diffraction of the read-out beam is calculated from (38). These calculations are the same as in the case without self-diffraction. To include the effects of self-diffraction it is, however, necessary to solve in addition the diffraction problem of the writing process. Therefore the next step is to calculate the effects of optical activity, field-induced birefringence and diffraction of the reference and writing beam from (37) and (38). In this way the intensities and polarization states of the reference and writing beams are altered, thereby altering the modulation depths and phases of the gratings in the next slab.

To illustrate how self-diffraction affects the grating inside the crystal, we have in Fig. 9(a) and (b) plotted the relative grating strength (modulation depth) and the position of the grating inside the crystal (given as the grating phase) for a typical diffraction problem. It is seen from Fig. 9(a) that the grating strength decreases slightly throughout the crystal and in addition exhibits an oscillatory behavior. The oscillations are also seen in the grating phase, see Fig. 9(b). If self-diffraction effects were neglected, the grating strength and grating phase would be constant throughout the crystal. However, due to self-diffraction and the anisotropic nature of the BSO crystal, the polarization states of the reference and writing beams change through the crystal, giving rise to distortions in the grating strength and phase.

When self-diffraction is included, the calculation time for the model is of course increased because two diffraction problems must be solved: in order to determine the gratings dynamically the diffraction problem during writing must first be solved, allowing in turn the solution of the actual diffraction problem during read-out. As shown above, both diffraction problems are solved simultaneously using the OBP method.

\section{E. Convergence}

The validity of our approximations is of course dependent on the number of slabs or, more precisely, on the slab thickness 


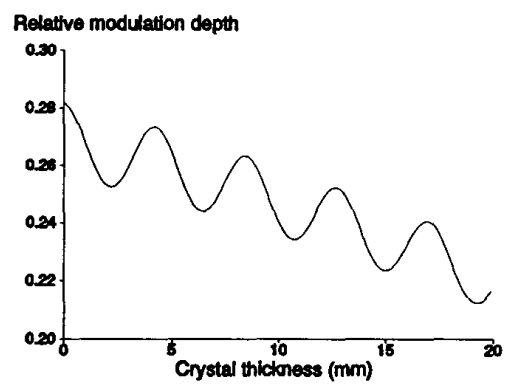

(a)

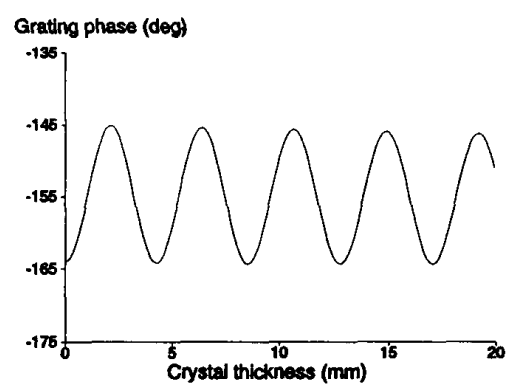

(b)

Fig. 9. Influence of self-diffraction in an autocorrelator setup. (a) Relative grating strength (modulation depth) versus the crystal thickness. (b) Grating phase versus crystal thickness. Parameters used in calculations: $I_{R e f}=1.45 \mathrm{~mW} / \mathrm{cm}^{2}, I_{o b j}=0.030 \mathrm{~mW} / \mathrm{cm}^{2}$, applied field $5.8 \mathrm{kV} / \mathrm{cm}$, and fringe spacing of $4.9 \mu \mathrm{m}$.

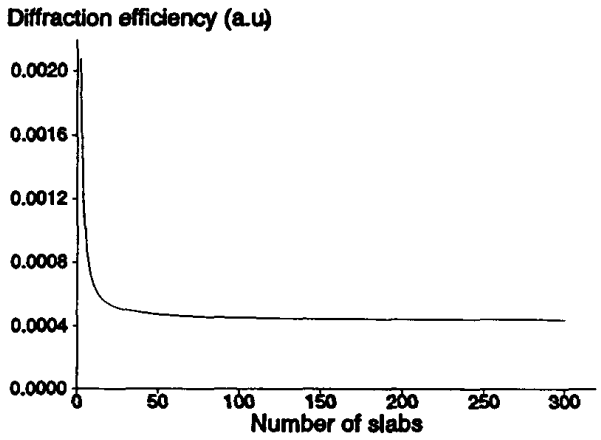

Fig. 10. Convergence of the diffraction efficiency (a.u.) versus the number of slabs.

$\Delta z$ compared to the diffraction efficiency. It turns out, however, that only a relatively small number of slabs is necessary to obtain an accurate solution to a typical diffraction problem. In Figs. 10-12 we have shown the diffraction efficiency, ellipticity (ratio of minor to major axis of the polarization ellipse) and polarization angle of the diffracted ray versus the number of slabs for a typical diffraction problem using a BSO crystal of $3 \mathrm{~mm}$ thickness, a grating period of $5 \mu \mathrm{m}$, an applied dc electrical field of $5 \mathrm{kV} / \mathrm{cm}$, and using a modulation depth of $m=0.30$. From Figs. $10-12$ it is clear that only $30-50$ slabs are necessary to obtain a good solution; using more than 100 slabs will not improve the solution significantly. The main reason for the fine convergence behavior of the algorithm is the fact that the phase of each ray is maintained throughout the calculations, thus taking into account birefringence, optical activity, and diffraction simultaneously.

The calculation time for solving this diffraction problem using 100 slabs is approximately $5 \mathrm{~s}$ using an AT286-type $12-\mathrm{MHz}$ personal computer with a numerical coprocessor, and approximately $0.5 \mathrm{~s}$ using an AT486-type $25 \mathrm{MHz}$ personal computer. If self-diffraction effects are included, the calculation time is increased to respectively 32 and $3 \mathrm{~s}$.

\section{EXPERIMENTS}

In this section we investigate experimentally the polarization properties and diffraction efficiency of the BSO autocorrelator

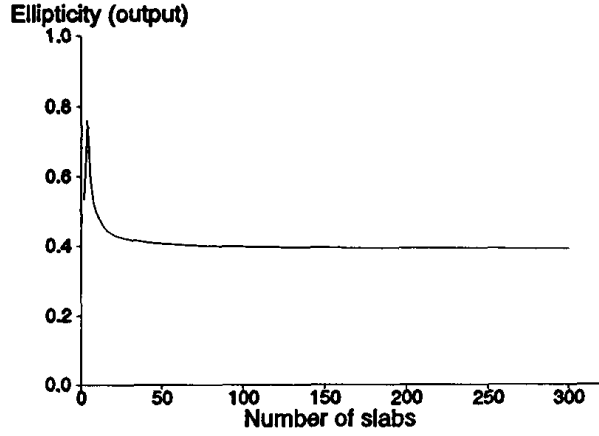

Fig. 11. Convergence of the ellipticity versus the number of slabs.

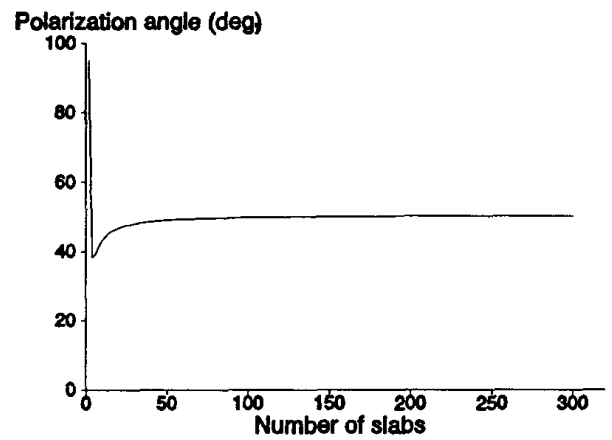

Fig. 12. Convergence of the angle of polarization versus the number of slabs.

and compare these results with results obtained with the OBP model. The experimental autocorrelator is shown in Fig. 13. The autocorrelation process is carried out in three steps: 1) Recording of the hologram in the BSO crystal (writing); 2) formation of the autocorrelation function (read out); and 3) removal of the holograms in the crystal (erasure).

The experiment corresponds to an autocorrelation of the simplest possible PIV image. Whereas a PIV image will normally contain a number of double-exposed particle images and in addition noise and incomplete pairs (single exposures), we consider the case of a single double-exposed particle image. This could be simulated by two pinholes with the proper 


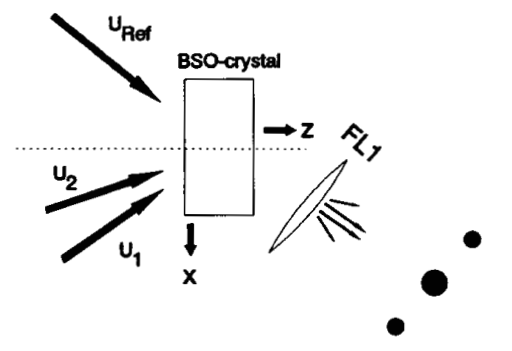

Fig. 13. Principle of the autocorrelator setup.

separation illuminated by the laser creating two spherical waves emanating from the pinholes. After the Fourier transform performed by lens $L_{1}$ [see Fig. 2(a) and Fig. 13], these spherical waves would be transformed into two plane waves with a small angular separation. To obtain better control of the experimental conditions we have chosen to provide as object waves to the BSO crystal two plane waves directly with a small adjustable angular separation $(\delta \theta)$ and with controlled polarization angle and ellipticity.

The two plane object waves $U_{1}$ and $U_{2}$ and the reference wave $U_{R e f}$ induce phase gratings in the photorefractive BSO crystal, see Fig. 13. There will be two gratings $G_{1}$ and $G_{2}$ caused by the interference of the reference wave with each of the two object waves, and one grating $G_{3}$ caused by interference between the two object waves. The autocorrelation function can be interpreted by considering the diffraction of the two object waves in these gratings during the read-out process: The center peak consists of two contributions corresponding to Bragg diffraction of the two object waves in their own gratings $G_{1}$ and $G_{2}$. The two side peaks correspond to off-Bragg diffraction of each object in the grating formed by the other object wave and the reference wave, i.e., diffraction of $U_{1}$ from $G_{2}$ and $U_{2}$ from $G_{1}$. In these considerations we have neglected diffraction in $G_{3}$ since the two object waves typically are much weaker than the reference wave. Furthermore we have assumed a linear superposition of the three gratings. In the numerical model the grating $G_{3}$ is included.

In these experiments we used an $\mathrm{Ar}^{+}$-ion laser at 514.5 $\mathrm{nm}$ (single longitudinal frequency) and a BSO sample with dimensions thickness $\times$ height $\times$ width $=3 \times 7 \times 6 \mathrm{~mm}^{3}$. The crystal is cut in the $\langle\overline{1} 10\rangle$ direction and has gold electrodes on the (110) faces, see Fig. 5. The autocorrelation function was detected with a CCD camera and analyzed on a personal computer with a frame grabber.

The polarization state of the diffracted beams was measured as a function of the polarization state of the incident beams. Furthermore, the polarization state of the output was measured as a function of the applied field across the crystal and of the angle $\delta \theta$ between the two object beams. The polarization state of the light beams is described by two parameters; the polarization angle $\psi$ and the ellipticity $e$. The polarization angle $\psi$ is the angle between the major axis of the polarization ellipse and the vertical, and the ellipticity is the ratio between the minor axis and the major axis:

$$
e=\frac{b}{a}, \quad|a| \geq|b|
$$

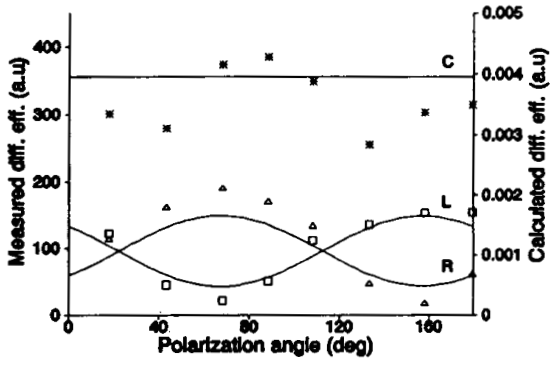

(a)

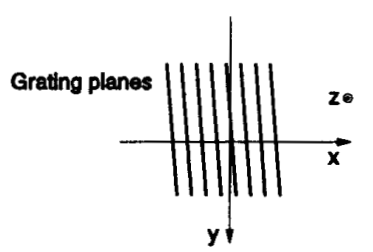

(b)

Fig. 14. (a) Diffraction efficiency (a.u.) of each of the three autocorrelation peaks versus the polarization angle of the incident light. The incident light is linearly polarized. The solid lines correspond to our calculations, and the markers to the measured data; ${ }^{*}$ center peak, $\square$ left peak, $\Delta$ right peak (other parameters in text). (b) Tilt of grating in crystal.

Negative values of $e$ correspond to left-circular light and positive values correspond to right-circular light.

\section{A. Results}

In Fig. 14(a) we have plotted the diffraction efficiency [in arbitrary units of the frame grabber (a.u.)] of each peak versus the polarization angle of the linearly polarized incident light. The applied field across the BSO crystal is $5.8 \mathrm{kV} / \mathrm{cm}$, the Bragg angle $\theta_{B}=2.2^{\circ}$, and the separation angle $\delta \theta$ between the two object beams is $0.049^{\circ}$. This separation angle corresponds to the displacement of the single particle during the double exposure. The intensities of the incident beams are $I_{R e f}=1.17 \mathrm{~mW} / \mathrm{cm}^{2}, I_{L}=0.031 \mathrm{~mW} / \mathrm{cm}^{2}$, and $I_{R}=0.036 \mathrm{~mW} / \mathrm{cm}^{2}$. The noise limit in our measurements of the diffraction efficiency is $\mathbf{2 0}$ (in arbitrary units of the frame grabber). We have measured the optical rotatory power for the BSO crystal and found $\rho=38.3^{\circ} \mathrm{mm}^{-1}$ at $514.5 \mathrm{~nm}$. The above parameters together with the parameters listed in Table I were used in our calculations. In Fig. 14(a) we obtain good agreement between calculations and measurements for the two side peaks, whereas the diffraction efficiency measurements of the center peak show a sinusoidal deviation. A sinusoidal behavior has been predicted by Mallick et al. [13] when the grating planes have a small tilt with respect to the (110) face of the photorefractive crystal, or the $x$-axis as shown in Fig. 14(b). Thus, if the crystal is mounted with a small tilt angle in the experimental setup, the diffraction efficiency of the center peak will exhibit a variation, as in Fig. 14(a). In our numerical model we have not included a tilt of the grating planes with respect to the vertical, and thus the sinusoidal behavior is not predicted by our model. A tilt of the grating planes can be included in our model by appropriate modification of the 


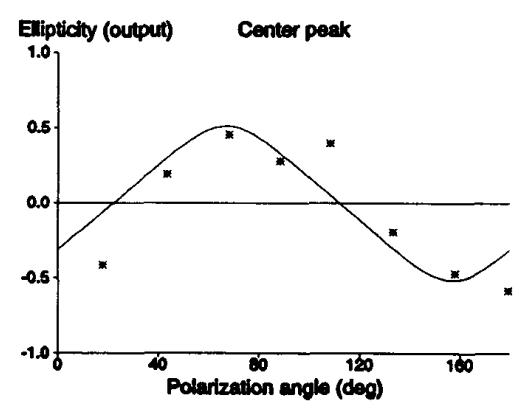

(a)

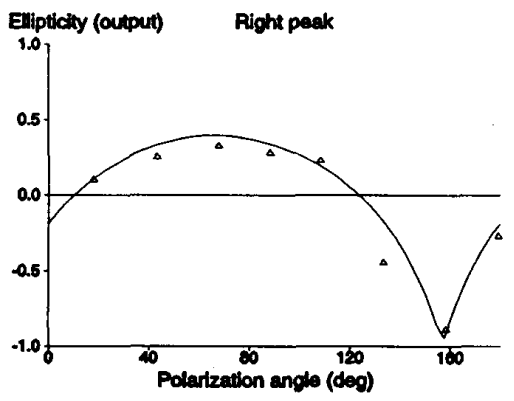

(c)

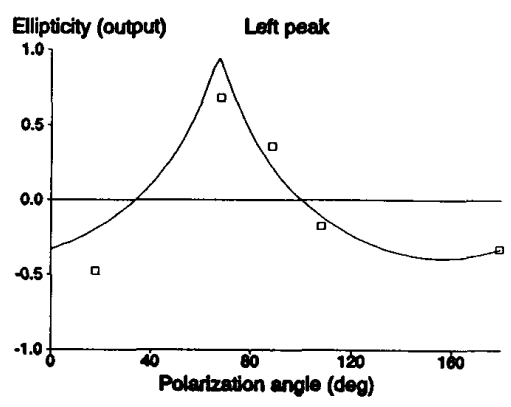

(b)

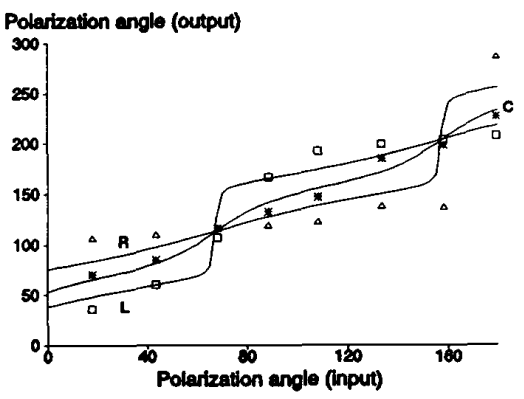

(d)

Fig. 15. (a)-(c) Ellipticity of each of the three autocorrelation peaks versus the polarization angle of the incident light. The incident light is linearly polarized. The solid lines correspond to our calculations, and the markers to the measured data. (a) * Center peak. (b) $\square$ Left peak. (c) $\Delta$ Right peak (other parameters in text). (d) Polarization angle of each of the three autocorrelation peaks versus the polarization angle of the incident light. The incident light is linearly polarized. The solid lines correspond to our calculations, and the markers to the measured data; * center peak, $\square$ left peak, $\triangle$ right peak (other parameters in text).

TABLE I

Physical Parameters for $\mathrm{Bi}_{12} \mathrm{SiO}_{20}$ aT $\lambda_{0}=514.5 \mathrm{~nm}$ Used in THE CALCULATIONS [16].

\begin{tabular}{ll}
$\alpha\left(\mathrm{cm}^{-1}\right)$ & 2 \\
& \\
$r_{41}(\mathrm{pm} / \mathrm{V})$ & 4.52 \\
$N_{A}\left(\mathrm{~cm}^{-3}\right)$ & $10^{16}$ \\
$\epsilon_{d c}$ & 56 \\
\hline
\end{tabular}

permittivity matrix. Another explanation for the deviation of the diffraction efficiency of the center peak can be found in self-diffraction, which will be discussed in Section III.B.

In Figs. 15(a)-(c) we have plotted the ellipticity of each of the three autocorrelation peaks versus the polarization angle of the incident beams for the same parameters mentioned above, and we obtain good agreement between calculations and measurements. In Fig. 15(d) we have plotted the polarization angle of each of the three autocorrelation peaks versus the polarization angle of the incident beams. Again we obtain good agreement between calculations and measurements. The ellipticity curves of the side peaks exhibit peaks located where the corresponding curves for the polarization angle exhibit jumps. Furthermore, when a peak appears in one of the side peaks, it is always located at the same polarization angle, and if both side peaks exhibit such behavior the locations are always approximately $90^{\circ}$ from each other. We shall return to this discussion later in this section.
The curves plotted in Figs. 14-15 show good agreement with out theoretical predictions. The incident beams, however, were always linearly polarized. In Fig. 16 we have plotted the polarization angle and ellipticity versus the polarization angle of the incident light, but now the ellipticity of the incident beams is changed to $e_{i}=0.5$, the applied field is $3 \mathrm{kV} / \mathrm{cm}$ and the intensities are now $I_{\text {Ref }}=1.47 \mathrm{~mW} / \mathrm{cm}^{2}, I_{L}=0.035$ $\mathrm{mW} / \mathrm{cm}^{2}$, and $I_{R}=0.042 \mathrm{~mW} / \mathrm{cm}^{2}$ (the modulation depths are unchanged). These curves also show good agreement between calculations and measurements. We see from Fig. 16 that only the left side peak exhibits a jump in polarization angle and a corresponding peak in ellipticity, whereas the right peak exhibits smooth curves.

\section{B. Discussion and Optimization}

In general, all our calculations exhibit good agreement with experiments, and therefore we consider our numerical model described in Section II as experimentally verified. In the numerical calculations we have not made any fit of physical parameters such as acceptor concentration $N_{A}$, electrooptic coefficient $r_{41}$ and absorption $\alpha$, and we have not made any fit of the experimental values such as applied field, separation angle, incident ellipticity, etc. System noise, for example scattered light, detector noise, etc., in the experimental setup may have an influence on some of the measurements, especially those close to the noise limit. Furthermore, in all of our calculations we have excluded self-diffraction effects, since we wanted to examine the influence of some physical parameters such as birefringence, optical rotatory power and separation angle $\delta \theta$. 


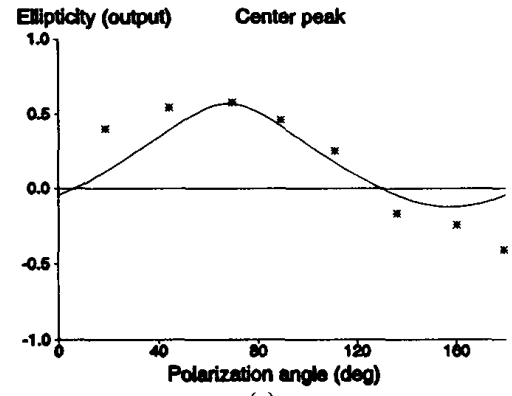

(a)

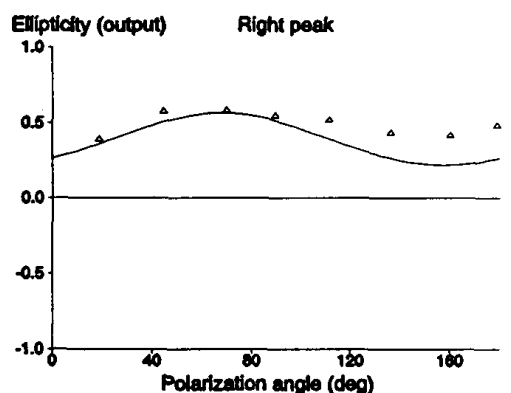

(c)

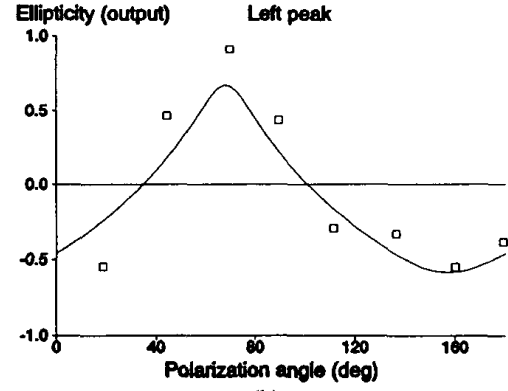

(b)

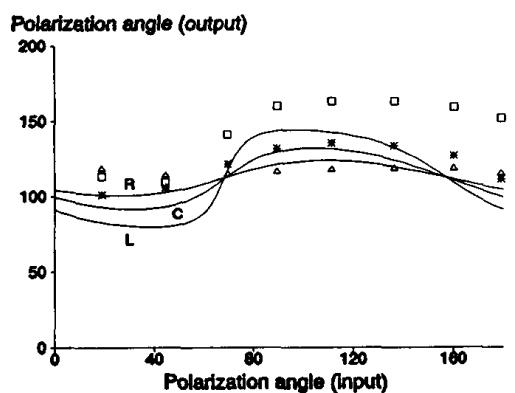

(d)

Fig. 16. (a)-(c) Ellipticity of each of the three autocorrelation peaks versus the polarization angle of the incident light. The incident light has the ellipticity $e_{i}=0.5$. The solid lines correspond to our calculations, and the markers to the measured data. (a) * Center peak. (b) $\square$ Left peak. (c) $\triangle$ Right peak (other parameters in text). (d) Polarization angle of each of the three autocorrelation peaks versus the polarization angle of the incident light. The incident light has the ellipticity $e_{i}=0.5$. The solid lines correspond to our calculations, and the markers to the measured data; $*$ center peak, $\square$ left peak, $\triangle$ right peak (other parameters in text).

In Figs. 17(a)-(d), however, we have plotted computed values of the diffraction efficiency and polarization angle versus the polarization angle of the incident light both excluding and including self-diffraction effects. These curves show that self-diffraction effects have a significant influence on the diffraction efficiency of the center peak, whereas the influence on the side peaks is less pronounced. The reason for this is that the center peaks consists of two diffraction contributions, as explained in Section III. This makes the center peak very sensitive to small variations in the phases of the gratings written by the two object beams and the reference beam. The reason that self-diffraction effects have a significant influence even though the diffraction efficiency is low is that in an autocorrelator setup the reference beam is very intense compared to the object beams. During the writing of the gratings the intense reference beam diffracts in the gratings inside the crystal whereby it adds to the weak object beams. In our setup the reference beam is 40 times more intense than the object beams, and consequently diffraction in the gratings can alter the object beams even though the diffraction efficiency is low. This in turn changes the gratings throughout the crystal as shown in Fig. 9, and as the center peak is very sensitive to the phases of the two gratings self-diffraction can have a significant impact on the diffraction efficiency of the center peak. Fig. 17 also shows that self-diffraction smooths out the jumps in the curves. Self-diffraction effects are subject for further numerical and experimental investigations.

As mentioned earlier, the curves for the polarization angle and the ellipticity of the two side peaks may exhibit jumps and peaks, and the location of these points is always $90^{\circ}$ displaced and always at the same incident angle independent of the separation angle, applied field, and incident polarization state. We have not yet been able to explain the physical reason for these extremes, but we have found that the locations of the extremes (if any) are determined by the optical rotatory power and the crystal thickness. In Fig. 18 we have plotted the calculated values for the ellipticity of the right side peak versus the incident polarizaion angle for various crystal thicknesses with the parameters used in Figs. 14-15. The incident beams are linearly polarized and we have excluded self-diffraction effects. We found that the polarization angle for the peaks moved approximately $\Delta \psi_{i}=0.5 \cdot \rho \cdot \Delta d$, were $\Delta d$ is the change in crystal thickness and $\rho$ is the optical rotatory power.

We have now demonstrated that the numerical model discussed in Section II agrees with experimental results, and that it is applicable for further investigations of the multibeam coupling in the BSO crystal. Also we used the model during optimization of the autocorrelator. In the following we will demonstrate the usefulness of the numerical model during optimization of the autocorrelator.

In an experimental setup the signal-to-noise ratio is very important for the performance of the autocorrelator [19]. As the center peak is very intense, its presence may degrade the performance of the autocorrelator, in particular with regard to detection of small particle displacements. It is therefore desirable to be able to suppress the center peak, especially as it does not contain any useful information. From our calculations based on a crystal length of $3 \mathrm{~mm}$, we have found that for a 

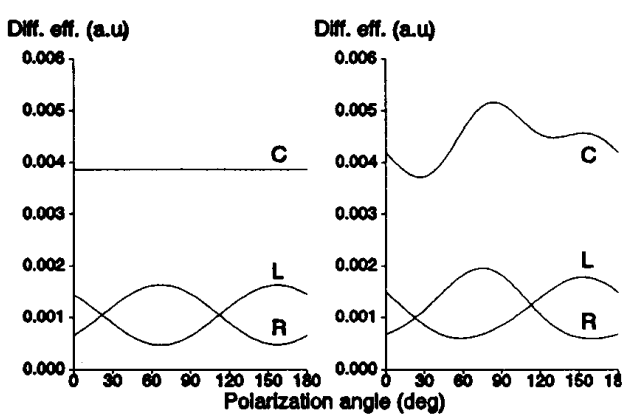

(a)

(b)

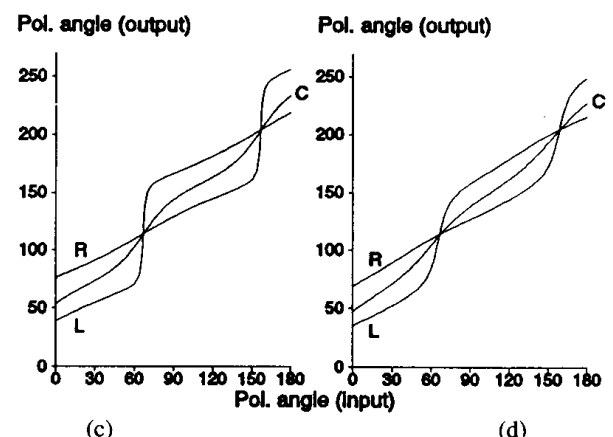

Fig. 17. (a)-(b) Diffraction efficiency (a.u.) of each of the three autocorrelation peaks versus the polarization angle of the incident light. The incident light is linearly polarized. C, L, and R correspond to the center, left, and right peak, respectively. (a) Self-diffraction effects excluded. (b) Self-diffraction effects included. (c)-(d) Polarization angle of each of the three autocorrelation peaks versus the polarization angle of the incident light. The incident light is linearly polarized. C, L and R correspond to the center, left, and right peak, respectively. (c) Self-diffraction effects excluded. (d) Self-diffraction effects included.

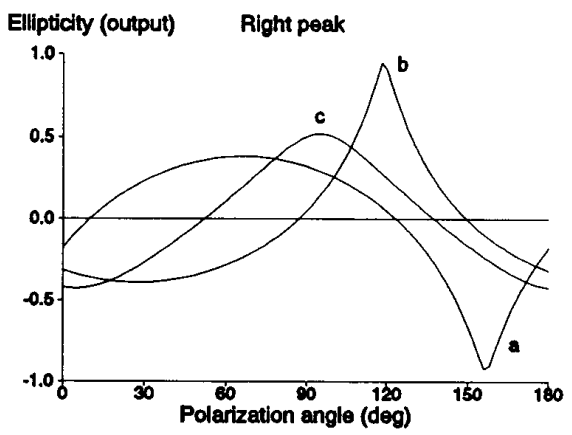

Fig. 18. Ellipticity of the right side peak versus polarization angle of the incident light. The incident light is linearly polarized, and self-diffraction effects are excluded. (a) Crystal thickness $3 \mathrm{~mm}$. (b) Crystal thickness $5 \mathrm{~mm}$. (c) Crystal thickness $6 \mathrm{~mm}$.

constant value of the applied field, the center peak is linearly polarized for linearly polarized incident light with polarization angles $\psi_{i} \approx 22^{\circ}$ or $\psi_{i} \approx 112^{\circ}$ independent of the separation angle $\delta \theta$. Furthermore, the polarization angle of the center peak does not vary with the angle $\delta \theta$, see Fig. 19(a). Thus, the center peak can be suppressed by placing a polarizer behind the BSO crystal, and the performance of the autocorrelator is enhanced provided that the side peaks are allowed to pass the polarizer. An additional problem has to be considered: The

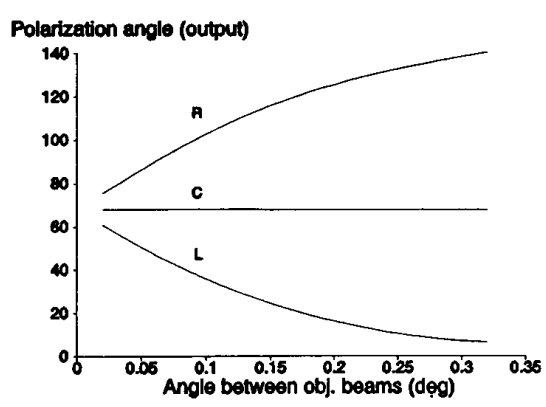

(a)

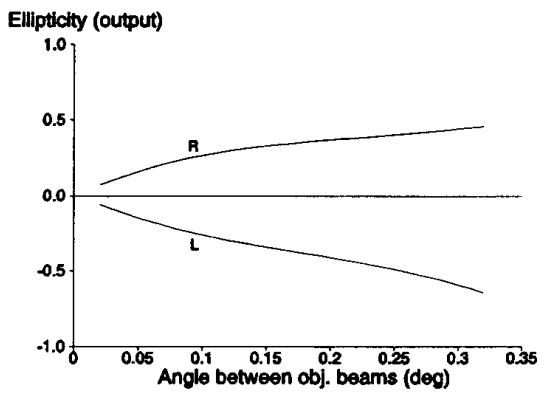

(b)

Fig. 19. (a) Polarization angle of the three correlation peaks versus the angle between the object beams $\delta \theta$. (b) Ellipticity of the two side peaks versus the angle between the object beams $\delta \theta$. The incident light is linearly polarized, with polarization angle $\psi_{i}=22.5^{\circ}$. Other parameters as in Fig. 14 .

polarization state of the side peaks varies with the angle $\delta \theta$, thus causing the signal-to-noise ratio to vary with the particle displacement. An example of this is shown in Fig. 19, showing the polarization angle and ellipticity versus the angle $\delta \theta$ with $\psi_{i}=22.5^{\circ}$, and the rest of the parameters as in Fig. 15 . However, in a typical correlator application the angular range for $\delta \theta$ would be from $0.0^{\circ}$ to $0.08^{\circ}$ for a focal length of the first lens of $100 \mathrm{~mm}$.

For some applications, choosing the proper crystal thickness is of great importance. In Fig. 20 we have shown the diffraction efficiency versus crystal thickness with an applied field of 5.8 $\mathrm{kV} / \mathrm{cm}, \psi_{i}=0^{\circ}, e_{i}=0$, and the rest of the parameters as in Fig. 15. It is obvious that the peak diffraction efficiency occurs at different crystal thicknesses. Therefore such curves are useful when choosing the best crystal thickness for optimization of the diffraction efficiency in a certain application. As is seen from Fig. 20, the diffraction efficiency for the two side peaks cannot be optimized at the same crystal thickness, since their oscillations are about $180^{\circ}$ out of phase. For a crystal thickness at approximately $4 \mathrm{~mm}$, it is seen that the diffraction efficiency of the center peak is at a minimum and the two side peaks are of equal strength, which could be of interest in an autocorrelator setup. It is also seen from Fig. 20 that the diffraction efficiency of the three correlation peaks increases with the crystal thickness until the point where absorption becomes dominant. When the crystal thickness exceeds 16 $\mathrm{mm}$ the two side peaks have almost vanished because of the absorption and the fact that the Bragg condition becomes more selective. 


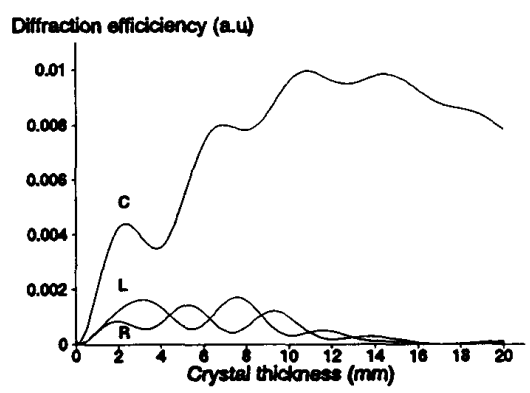

Fig. 20. Diffraction efficiency (a.u.) of the three side peaks versus crystal thickness, with other parameters as in Fig. 15.

The complicated polarization behavior described in this section is a consequence of the optical activity and fieldinduced birefringence of the BSO crystal. The two side peaks, which correspond to off-Bragg diffraction, exhibit a behavior that differs from that of the center peak (which corresponds to Bragg diffraction). Furthermore, the fact that the two side peaks behave differently is a consequence of off-Bragg diffraction.

\section{The Bias Problem}

In our special application of the correlator as a processor for particle image velocimetry recordings, we have to be concerned about the performance of the correlator for different values of the particle image displacement. A special situation occurs in practice in measurements of turbulent velocity fields. The PIV recording may then contain particle image displacements ranging from near zero to near the dimensions of the interrogation area corresponding to different values of the velocity over the entire recorded field. Moreover the velocities may have different directions resulting in image displacements of different orientations with respect to the applied field. These variations in image displacement cause the holographic gratings in the BSO crystal to have differing spatial frequencies and directions. Since the diffraction efficiency and the output polarization depend on the fringe spacing and orientation, we might expect variations in the sensitivity of the crystal as a processor for different values of the velocity vector. The effect may result in a phenomenon called velocity bias, i.e., distortions in the measured velocity distribution caused by the varying sensitivity of the crystal to different velocity values. The phenomenon may occur in at least two different situations: In the first, data may be predominantly missed in regions of the PIV recording, where the velocity is such that the signal-to-noise ratio is bad or the diffraction efficiency is low. In the other case, the crystal is used in a mode, where a large interrogation area containing a statistical ensemble of velocities is processed simultaneously (see Jakobsen and Buchhave [20]). In this case some particular velocity values may be predominantly missed.

Our simple model of the correlator may be used to illustrate the problem. As we have seen the correlation side peaks may be understood as diffraction of one object beam (corresponding to one position of the particle image) in the grating introduced by the other object beam (corresponding to the other particle image). Thus we have an example of off-Bragg diffraction, and the efficiency of the correlator can be understood as the off-Bragg diffraction efficiency. Different image displacements may then give different side peak intensities and thereby introduce bias. As a first estimate for this effect we may use the width of the Bragg angle range in a thick grating. We then find that the displacement $\Delta X$ should fulfill the following condition:

$$
\Delta X<\frac{\lambda f}{2 d \sin \left(\theta_{B}\right)}, \quad \text { or } \quad \Delta \theta<\frac{\lambda}{2 d \sin \left(\theta_{B}\right)}
$$

In our case the Bragg angle $\theta_{B}$ is about $3^{\circ}$ and the crystal thickness $d$ is $3 \mathrm{~mm}$. We then find that the maximum allowable displacement is about $150 \mu \mathrm{m}$ with a focal length of the first Fourier transforming lens of $100 \mathrm{~mm}$. This is a displacement, which may very well be found in practice in an interrogation area of $1 \mathrm{~mm}^{2}$.

The correlation center peak, on the other hand, may be understood as the diffraction of each object beam in its own grating with the reference beam. Thus in principle the intensity of the center peak and the polarization of the read-out beam should not depend on the image displacement. This is actually borne out by both our experiments and our numerical model. Even relatively large changes in the detuning angles of the input beams do not cause any appreciable change in the input angle for which the output is linearly polarized. Neither is the diffraction efficiency of the center peak sensitive to small angular changes in the writing beams.

As a final comment it may be mentioned that the recording of the particle images may be modified by a technique known as image shifting [21]. Using this technique a displacement bias or offset is deliberately introduced in the recording by shifting the image between the two exposures of the film resulting in a smaller relative variation in the magnitude and direction of the image displacement at the cost of a corresponding loss of resolution in the velocity determination. Application of such an image shifting technique may reduce the bias problem discussed above.

\section{CONCLUSIONS}

We have presented a numerical model of a BSO optical autocorrelator. The model is based on the beam propagation method and includes multiple gratings effects, optical activity, and birefringence in the BSO crystal. The model extends previous numerical models. It keeps track of the phases of all interacting beams in the BSO crystal and consequently includes self-diffraction. Furthermore, it is valid for multibeam interaction, which is not symmetric about the axis of propagation.

The numerical model is compared with an experimental investigation of the polarization properties of a BSO autocorrelator. In general we obtain an asymmetric autocorrelation function with different polarization states of the autocorrelation peaks. The polarization states of the peaks are sensitive to parameters such as applied voltage on the BSO crystal, the crystal thickness, and the displacement of the particles. We obtain good agreement between the experimental results and the numerical model, and we show how the model is used to 
optimize the performance of the BSO autocorrelator. Finally it is shown that self-diffraction effects are important even in the limit of low diffraction efficiencies, where self-diffraction usually is neglected.

\section{REFERENCES}

[1] R. J. Adrian, "Particle imaging techniques for experimental fluid mechanics," Ann. Rev. Fluid Mech., vol. 23, pp. 261-304, 1991.

[2] P. Buchhave, "Particle image velocimetry-status and trends," in Proc. 2nd World Conf. on Experimental Heat Transfer, Fluid Mechanics and Thermodynamics, June 1991, Dubrovnik, Yugoslavia, J. F. Keffer, R. K. Shah, and E. N. Ganic, Eds.; Elsevier, 1991, pp. 35-47.

[3] R. J. Adrian, "Statistical properties of particle image velocimetry measurements in turbulent flow," in Laser Anemometry in Fluid Mechanics III, eds. R. J. Adrian, D. F. G. Durao, F. Durist, and J. H. Whitelaw, LADOAN, Instituto Superior Technico, Lisbon, pp. 115-129, 1988.

[4] J. M. Coupland and C. J. D. Pickering, "Particle image velocimetry: estimation of measurement confidence in low seeding densities," Opt. and Lasers in Eng., vol. 9, pp. 201-210, 1988.

[5] P. Buchhave and M. L. Jakobsen, "Optical correlator for PIV image processing," in Laser Anemometry, Advances and Applications, Proc. 3rd Int. Conf., BHRA. Springer-Verlag, 1990, pp. 609-616.

[6] P. Buchhave, M. L. Jakobsen, C. H. Westergaard, and J. N. Soerensen "PIV: measurement of the early transition of a rotating flow in a closed cylinder," in Proc. 4th Int. Conf. on Laser Anemometry-Advances and Appl., Cleveland, Ohio, August 1991, pp. 45-51.

[7] H. Kogelnik, "Coupled wave theory for thick hologram gratings," Bell Syst. Tech. J., vol. 48, pp. 2909-2947, 1969.

[8] M. P. Petrov, T. G. Pencheva, and S. I. Stephanov, "Light diffraction from volume phase holograms in electrooptic photorefractive crystals," J. Opt. (Paris) vol. 12, pp. 287-292, 1981.

[9] T. G. Pencheva, M. P. Petrov, and S. I. Stephanov, "Selective properties of volume phase holograms in photorefractive crystals," Opt. Commun. vol. 40, pp. 175-178, 1982.

[10] N. V. Kuhktarev, G. E. Dovgalenko, and V. N. Starkov, "Influence of the optical activity on hologram formation in photorefractive crystals," Applied Phys. A., vol. 33, pp. 227-230, 1984.

[11] F. Vachss and L. Hesselink, "Holographic beam coupling in anisotropic photorefractive media," J. Opt. Soc. Am. A., vol. 4, pp. 325-339, 1987.

[12] E. N. Glytsis and T. K. Gaylord, "Three-dimensional (vector) rigorous coupled-wave analysis of anisotropic grating diffraction," J. Opt. Soc. Am. A., vol. 7, pp. 1399-1420, 1990.

[13] S. Mallick, D. Rouède, and A. G. Apostolidis, "Efficiency and polarisation characteristics of photorefractive diffraction in a $\mathrm{Bi}_{12} \mathrm{SiO}_{20}$ crystal," J. Opt. Soc. Am., B., vol. 4, pp. 1247-1259, 1987.

[14] R. V. Johnson and A. R. Tanguay, Jr., "Optical beam propagation method for birefringent phase grating diffraction," Opt. Eng., vol. 25, no. 2 , pp. $235-249,1986$

[15] A. Marrakchi, R. V. Johnson, and A. R. Tanguay, Jr., "Polarisation properties of photorefractive diffraction in electrooptic and optically active sillenite crystals (Bragg regime)," J. Opt. Soc. Am., B, vol. 3, pp. 321-336, 1986

[16] A. Marrakchi, R. V. Johnson, and A. R. Tanguay, Jr., "Polarisation properties of enhanced self-diffraction in sillenite crystals," IEEE $J$. Quantum Electron., vol. QE-23, pp. 2142-2151, 1987.

[17] T. J. Hall, R. Jaura, L. M. Conners, and P. D. Foote, "The photorefractive effect - a review," Prog. Quant. Electr., vol. 10, pp. 77-146, 1985

[18] A. Yariv and P. Yeh, Optical Waves in Crystals. New York: Wiley, 1984.

[19] P. E. Andersen, A. Marrakchi, P. M. Petersen, P. Buchhave, and B. Edvold, "Photorefractive particle image velocimetry: performance enhancement using the polarization properties of BSO crystals," Techn. Dig. on Photorefractive Materials, Effects and Devices, Optical Society of America, Washington, DC, 1991, vol. 14, paper PD3.

[20] M. L. Jakobsen and P. Buchhave, "PIV-processing: Parallel processing with optical correlators," Proc. 5th Int. Symp. on Applications of Laser Techn. to Fluid Mechanics, July 1990, Lisbon, Portugal.
[21] R. J. Adrian, "An image shifting technique to resolve directional ambiguity in double-pulsed velocimetry," Appl. Optics, vol. 25, pp. 3855-3858, 1986.

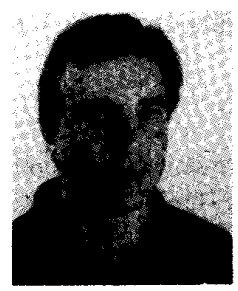

Bent Edvold was born on December 18, 1967, in Copenhagen, Denmark. He received the M.Sc. degree in electrical engineering in 1991 from the Technical University of Denmark. He is presently pursuing the Ph.D., also at the Technical University of Denmark, where he is researching optical fibers in LYCOM A/S

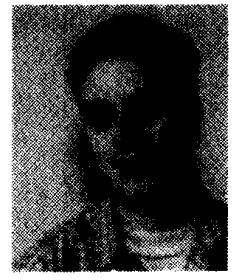

Peter E. Andersen was born on March 14, 1967, in Holback, Denmark. He received the M.Sc. degree in electrical engineering from the Technical University of Denmark in 1991. He is currently with the Physics Department oat the Technical University of Denmark, where he is pursuing the Ph.D. degree in nonlinear optics in photorefractive materials for dynamic optical interconnects.

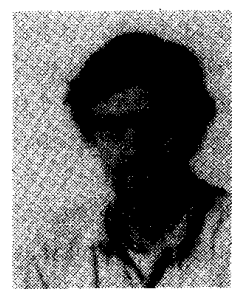

Preben Buchhave received the M.S. degree in physics from the Technical University of Denmark in 1964 and the Ph.D. from SUNY Buffalo in 1979.

From 1966 to 1970 he worked at Westinghouse R\&D Center's Quantum Optics Department. In 1971 he joined Dantec Electronics, where he worked on laser-based instumentation for fluid flow and particle diagnostics. After serving as a manager of research and development at Dantec, he was vice president of the Scientific Research and Equipment Division at the Allendale, NJ, division. Since 1988 he has been with the Technical University of Denmark, where he has been working with optical processing of image data. he is presently working with the nonlinear effects in photorefractives and in diode-pumped optical parametric oscillators.

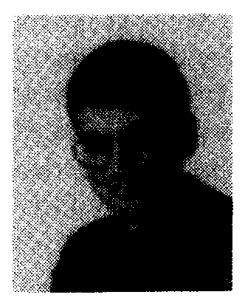

Paul M. Petersen received the M.Sc. degree in engineering and the Ph.D. degree from the Technical University of Denmark in 1983 and 1986, respectively.

Since 1989 he has been working with nonlinear optics in photorefractive materials for applications in optical signal processing. he has published numerous papers in the fields of optical phase conjugation, photorefractive oscillators, and four-wave mixing in nonlinear materials. $\mathrm{He}$ is now with the Optics and Fluid Dynamics Department at Risoe National Laboratory, Denmark. 